

LLNL-TR-435033

Isotopic Abundances and

Ratios in Arsenic Irradiated by

High-Energy Neutrons

W. E. Parker and J. M. Hall

Lawrence Livermore National Laboratory

P.O. Box 808, Livermore CA 94550

June 11, 2010 


\section{Disclaimer}

This document was prepared as an account of work sponsored by an agency of the United States government. Neither the United States government nor Lawrence Livermore National Security, LLC, nor any of their employees makes any warranty, expressed or implied, or assumes any legal liability or responsibility for the accuracy, completeness, or usefulness of any information, apparatus, product, or process disclosed, or represents that its use would not infringe privately owned rights. Reference herein to any specific commercial product, process, or service by trade name, trademark, manufacturer, or otherwise does not necessarily constitute or imply its endorsement, recommendation, or favoring by the United States government or Lawrence Livermore National Security, LLC. The views and opinions of authors expressed herein do not necessarily state or reflect those of the United States government or Lawrence Livermore National Security, LLC, and shall not be used for advertising or product endorsement purposes.

\section{Auspices Statement}

This work performed under the auspices of the U.S. Department of Energy by Lawrence Livermore National Laboratory under Contract DE-AC52-07NA27344. 


\title{
Isotopic Abundances and Ratios in Arsenic Irradiated by High-Energy Neutrons
}

\author{
W. E. Parker and J. M. Hall \\ Lawrence Livermore National Laboratory, P.O. Box 808, Livermore CA 94550
}

\begin{abstract}
This document provides derivations of the ${ }^{73} \mathrm{As},{ }^{74} \mathrm{As}$ and ${ }^{75} \mathrm{As}$ isotopic abundances and ratios in an arsenic sample irradiated by high-energy $(14 \mathrm{MeV})$ neutrons for $0 \leq t \leq \mathrm{T}$, where $\mathrm{T}$ is short compared to the natural decay times of the reaction products $\left(t_{1 / 2}\left({ }^{73} \mathrm{As}\right)\right.$ $\left.\sim 80.3 \mathrm{~d}, t_{1 / 2}\left({ }^{74} \mathrm{As}\right) \sim 17.8 \mathrm{~d}\right)$. The document also outlines the historic approach used to analyze arsenic data from experiments.
\end{abstract}

\section{Introduction}

The fusion energy production in a nuclear device was historically monitored by adding radiochemical detectors at various locations in the device during pre-shot work. These "detectors" were usually composed of small quantities of materials with relatively large $(\mathrm{n}, 2 \mathrm{n})$ reaction cross sections that could be used to estimate average neutron fluence at shot time. A variety of rare elements were used for this purpose including solid yttrium, zirconium, europium, bismuth, thulium and lutetium and gaseous xenon, krypton, argon and arsenic (usually in the form of arsine gas, $\mathrm{AsH}_{3}$ ), with the radiochemical products of the $(n, 2 n)$ and double $(n, 2 n)$ reactions from samples of the debris being measured after the shot was fired. The National Ignition Facility (NIF) will also use radiochemical diagnostics loaded in the NIF capsule (in particular, arsine gas) to estimate neutron fluences during and after the ignition process.

Because $(n, 2 n)$ reactions have high neutron-energy thresholds, the post-irradiation radiochemical diagnostic ratios are related to the high-energy neutron production. In the case of an arsenic radiochemical detector, the loaded detector material would be $100 \%{ }^{75} \mathrm{As}$. The ${ }^{75}$ As can undergo an (n,2n) reaction to produce ${ }^{74} \mathrm{As}$ ( $\sim 17.78$ day half-life) and the ${ }^{74}$ As can undergo an additional reaction with a second neutron to produce ${ }^{73}$ As $(\sim 80.3$ day half-life). In this case, the experimentally measured post-irradiation ratio of interest will be ${ }^{73} \mathrm{As} /{ }^{74} \mathrm{As}$. This radiochemical ratio is a measure of the fusion energy production, but its value will also depend on the energies of the neutrons involved since these reactions have specific energy thresholds below which they will not occur:

$$
\begin{aligned}
& { }^{75} \mathrm{As}+\mathrm{n} \rightarrow{ }^{74} \mathrm{As}+2 \mathrm{n} \text {; neutron energy threshold } \sim 11 \mathrm{MeV}, \\
& { }^{74} \mathrm{As}+\mathrm{n} \rightarrow{ }^{73} \mathrm{As}+2 \mathrm{n} \text {; neutron energy threshold } \sim 8.1 \mathrm{MeV} .
\end{aligned}
$$

Measuring a second order ratio such as ${ }^{73} \mathrm{As} /{ }^{74} \mathrm{As}$ allows a probe of the post-irradiation debris that does not depend upon the collection efficiency for the experiment, whereas measuring a first order ratio such as ${ }^{74} \mathrm{As} /{ }^{75} \mathrm{As}$ (loaded), requires that the collection effi- 
ciency for the particular experiment be determined. The collection efficiency is the fraction of the total post-irradiation debris that is extracted or collected from the experiment for post-irradiation diagnostics measurements.

It is important to note that, in addition to the material deliberately loaded into a device or a capsule during pre-shot work, elemental arsenic can appear elsewhere in the experimental environment, as arsenic is normally found in parts-per-million quantities in steel, lead and aluminum. The experimentally measured ${ }^{73} \mathrm{As} /{ }^{74} \mathrm{As}$ must, therefore, be corrected for the contribution of these background materials. If (as is usually the case) the mass of the loaded detector is relatively small and/or the surrounding experimental structures contain significant quantities of arsenic, this correction could be significant.

This document presents derivations of the relative isotopic abundances and ratios for the case of an arsenic radiochemical detector irradiated by $14 \mathrm{MeV}$ neutrons along with a description of the historical approach used to analyze data from experiments.

\section{Isotopic abundances and ratios}

When an arsenic sample with a natural abundance of $100 \%{ }^{75} \mathrm{As}$ is irradiated with highenergy neutrons, nuclear reactions such as ${ }^{75} \mathrm{As}(\mathrm{n}, 2 \mathrm{n}){ }^{74} \mathrm{As}$ and ${ }^{74} \mathrm{As}(\mathrm{n}, 2 \mathrm{n}){ }^{73} \mathrm{As}$ can be expected to occur with predictable rates $\lambda_{1}$ and $\lambda_{2}$, respectively:

$$
{ }^{75} A s \stackrel{\lambda_{1}=f\left(\text { nflux }, \sigma_{1}(n, 2 n)\right)}{\longrightarrow}{ }^{74} A s \stackrel{\lambda_{2}=f\left(\text { nflux }, \sigma_{2}(n, 2 n)\right)}{\longrightarrow}{ }^{73} A s .
$$

In this derivation we will assume that the $(n, 2 n)$ destruction rate for ${ }^{75}$ As is the same as that for ${ }^{74}$ As (i.e. $\lambda_{1} \sim \lambda_{2} \equiv \lambda$ ). As shown in Figure 1 at the end of this document, for arsenic irradiated by $14 \mathrm{MeV}$ neutrons, this approximation should be good to within $\sim 5 \%{ }^{1}$ We will also assume that the incident neutron flux can be treated as a constant for $0 \leq t \leq$ $\mathrm{T}$, where $\mathrm{T}$ is short compared to the natural decay times of the reaction products.

The number of ${ }^{75}$ As atoms present in the sample at a given time $0 \leq t \leq \mathrm{T}, n_{5}(t)$, will depend on the rate at which they are being destroyed in ${ }^{75} \mathrm{As}(\mathrm{n}, 2 \mathrm{n}){ }^{74} \mathrm{As}$ reactions. The rate of change in $n_{5}$ can be expressed as

$$
\frac{d n_{5}}{d t}=-\lambda n_{5}
$$

The number of ${ }^{74}$ As atoms present in the sample at time $t, n_{4}(t)$, will depend on both the rate at which they are being destroyed in ${ }^{74} \mathrm{As}(\mathrm{n}, 2 \mathrm{n}){ }^{73} \mathrm{As}$ reactions and the rate at which they are being created in ${ }^{75} \mathrm{As}(\mathrm{n}, 2 \mathrm{n}){ }^{74} \mathrm{As}$ reactions (i.e. the rate at which ${ }^{75} \mathrm{As}$ atoms are being destroyed). If we neglect the comparatively slow natural decay rate of ${ }^{74} \mathrm{As}\left(t_{1 / 2} \sim\right.$ $17.8 \mathrm{~d}$ ), the rate of change in $n_{4}$ can be expressed as

$$
\frac{d n_{4}}{d t}=-\lambda n_{4}+\lambda n_{5}
$$

\footnotetext{
${ }^{1}$ An analogous development for situations in which $\lambda_{1}$ cannot assumed to be equal to $\lambda_{2}$ (e.g. Monte Carlo simulations in which the full neutron spectrum is taken into account) will be outlined in Appendix A.
} 
Finally, the number of ${ }^{73}$ As atoms present in the sample at time $t, n_{3}(t)$, will depend on the rate at which they are being created in ${ }^{74} \mathrm{As}(\mathrm{n}, 2 \mathrm{n}){ }^{73} \mathrm{As}$ reactions (i.e. the rate at which ${ }^{74} \mathrm{As}$ atoms are being destroyed). If we neglect the comparatively slow natural decay rate of ${ }^{73} \mathrm{As}\left(t_{1 / 2} \sim 80.3 \mathrm{~d}\right)$, the rate of change in $n_{3}$ can be expressed as

$$
\frac{d n_{3}}{d t}=+\lambda n_{4}
$$

If $\lambda$ is assumed to be a constant, Eqn. (1) can easily be solved for $n_{5}(t)$ by direct integration; however, since we will be using differential operator techniques to solve for $n_{4}(t)$ in the next step, we will use the same approach to solve for $n_{5}(t)$ here in order to introduce the basic concepts to readers who might not be familiar with them. We will begin by rewriting Eqn. (1) in terms of the differential operator $D \equiv d / d t$ :

$$
\begin{aligned}
& \frac{d n_{5}}{d t} \equiv D n_{5}=-\lambda n_{5} \\
& \Rightarrow(D+\lambda) n_{5}=0 .
\end{aligned}
$$

The so-called "auxiliary equation" for Eqn. (4b) is $(m+\lambda)=0$, which has a unique real root $-\lambda$. Based on differential operator prescriptions ( $c f$. CRC math handbook), the general solution for $n_{5}$ will thus be of the form

$$
n_{5}(t)=c_{1} e^{-\lambda t} .
$$

Since the initial amount of ${ }^{75}$ As in the sample will be $n_{5}(0)$, the arbitrary constant $c_{1}$ in Eqn. (4b) must equal $n_{5}(0)$. The final solution for $n_{5}$ thus becomes

$$
n_{5}(t)=n_{5}(0) e^{-\lambda t} \text {. }
$$

While perhaps obvious, Eqn. (6) can be validated for arbitrary times $0 \leq t \leq \mathrm{T}$ by simply taking its derivative with respect to $t$ and comparing the result to the RHS of the original rate equation for $n_{5}$ (Eqn. (1)):

$$
\frac{d n_{5}}{d t}=-n_{5}(0) \lambda e^{-\lambda t}=-\lambda n_{5} \cdot(\sqrt{ })
$$

If we substitute the solution for $n_{5}(t)$ (Eqn. (6)) into Eqn. (2), we obtain

$$
\frac{d n_{4}}{d t}=-\lambda n_{4}+\lambda n_{5}(0) e^{-\lambda t}
$$

Equation (8) thus becomes a first order inhomogeneous differential equation which can be solved using differential operator techniques:

$$
\begin{gathered}
\frac{d n_{4}}{d t} \equiv D n_{4}=-\lambda n_{4}+\lambda n_{5}(0) e^{-\lambda t} \\
\Rightarrow(D+\lambda) n_{4}=\lambda n_{5}(0) e^{-\lambda t}
\end{gathered}
$$

Since the inhomogeneity on the RHS of Eqn. (9b) is proportional to $e^{-\lambda t}$ (analogous to Eqn. (5)), it must be a particular solution to a homogeneous differential equation of the form $(D+\lambda) r=0$ for some initial condition $r(0)=\lambda n_{5}(0)$, i.e., 


$$
r_{p}(t)=\lambda n_{5}(0) e^{-\lambda t}
$$

The relevance of this particular solution for the function $r$ becomes apparent when we apply the differential operator $(D+\lambda)$ to both sides of Eqn. $(9 \mathrm{~b})$ to obtain

$$
(D+\lambda)(D+\lambda) n_{4}=(D+\lambda) \lambda n_{5}(0) e^{-\lambda t}=(D+\lambda) r_{p}=0 \text {. }
$$

We now have a second order homogeneous differential equation for $n_{4}$ whose auxiliary equation, $(m+\lambda)(m+\lambda)=0$, has repeated real roots of $-\lambda$. Based on differential operator prescriptions, the general solution for $n_{4}$ will thus be of the form

$$
n_{4}(t)=c_{2} e^{-\lambda t}+c_{3} t e^{-\lambda t} \text {. }
$$

The arbitrary constants in Eqn. (12) can be resolved by reviewing the initial conditions for $n_{4}$ and its rate of change (Eqn. (2)). Since the natural abundance of arsenic is $100 \%$ ${ }^{75} \mathrm{As}$, the initial amount of ${ }^{74} \mathrm{As}, n_{4}(0)$, will be 0 and its initial rate of change (Eqn. (2) evaluated at $t=0$ ) will be $\lambda n_{5}(0)$. This means that the constants $c_{2}$ and $c_{3}$ must equal 0 and $\lambda n_{5}(0)$, respectively. The final solution for $n_{4}$ thus becomes

$$
n_{4}(t)=n_{5}(0) \lambda t e^{-\lambda t} .
$$

Equation (13) can be validated for arbitrary times $0 \leq t \leq \mathrm{T}$ by taking its derivative and comparing the result to the original rate equation for $n_{4}$ (Eqn. (2)):

$$
\frac{d n_{4}}{d t}=-n_{5}(0) \lambda^{2} t e^{-\lambda t}+n_{5}(0) \lambda e^{-\lambda t}=-\lambda n_{4}+\lambda n_{5} \cdot(\sqrt{ })
$$

If we substitute the solution for $n_{4}(t)$ (Eqn. (13)) into Eqn. (3), we obtain

$$
\frac{d n_{3}}{d t}=+\lambda n_{5}(0) \lambda t e^{-\lambda t}
$$

which can be solved by direct integration ( $c f$. CRC math handbook):

$$
\begin{gathered}
\int_{n_{3}(0)}^{n_{3}(t)} d n_{3}=n_{5}(0) \lambda^{2} \int_{0}^{t} u e^{-\lambda u} d u=n_{5}(0) \lambda^{2}\left[\frac{e^{-\lambda u}}{\lambda^{2}}(-\lambda u-1)\right]_{0}^{t} \\
\Rightarrow n_{3}(t)-n_{3}(0)=n_{5}(0)\left(1-e^{-\lambda t}-\lambda t e^{-\lambda t}\right) .
\end{gathered}
$$

Keeping in mind that that the initial amount of ${ }^{73} \mathrm{As}, n_{3}(0)$, will also be 0 , the final solution for $n_{3}$ thus becomes

$$
n_{3}(t)=n_{5}(0)\left(1-e^{-\lambda t}-\lambda t e^{-\lambda t}\right) .
$$

Equation (17) can be validated for arbitrary times $0 \leq t \leq \mathrm{T}$ by taking its derivative and comparing the result to the original rate equation for $n_{3}$ (Eqn. (3)):

$$
\begin{aligned}
\frac{d n_{3}}{d t} & =+n_{5}(0) \lambda e^{-\lambda t}-n_{5}(0) \lambda e^{-\lambda t}+n_{5}(0) \lambda^{2} t e^{-\lambda t} \\
& =+n_{5}(0) \lambda^{2} t e^{-\lambda t}=+\lambda n_{4} \cdot(\sqrt{ })
\end{aligned}
$$


In summary then, the validated solutions for the isotopic abundances of ${ }^{73} \mathrm{As},{ }^{74} \mathrm{As}$ and ${ }^{75}$ As for arbitrary times $0 \leq t \leq \mathrm{T}$ are

$$
\begin{gathered}
n_{3}(t)=n_{5}(0)\left(1-e^{-\lambda t}-\lambda t e^{-\lambda t}\right) \approx n_{5}(0) \frac{(\lambda t)^{2}}{2}(\lambda t \ll 1), \\
n_{4}(t)=n_{5}(0) \lambda t e^{-\lambda t} \approx n_{5}(0) \lambda t(\lambda t<<1)
\end{gathered}
$$

and

$$
n_{5}(t)=n_{5}(0) e^{-\lambda t} \approx n_{5}(0)(1-\lambda t)(\lambda t<<1),
$$

respectively. Note that $n_{3}(t)+n_{4}(t)+n_{5}(t)=n_{5}(0)$ for $0 \leq t \leq \mathrm{T}$ as we would expect (i.e. the exact solutions are self consistent and the approximate solutions for $\lambda t<<1$ are self consistent to within terms $\left.\sim(\lambda t)^{2}\right)$. Generic (dimensionless) plots of these isotopic abundances and the relative errors associated with using the approximate $(\lambda t<<1)$ solutions are shown in Figures $2 \mathrm{a}$ and $2 \mathrm{~b}$, respectively, at the end of this document for $\lambda t$ values ranging from 0 to 0.250 .

Using the solution set summarized above, the ${ }^{73} \mathrm{As} /{ }^{74} \mathrm{As}$ and ${ }^{74} \mathrm{As} /{ }^{75} \mathrm{As}$ isotopic ratios for arbitrary times $0 \leq t \leq \mathrm{T}$ become

$$
\frac{n_{3}(t)}{n_{4}(t)}=\frac{n_{5}(0)\left(1-e^{-\lambda t}-\lambda t e^{-\lambda t}\right)}{n_{5}(0) \lambda t e^{-\lambda t}}=\frac{\left(e^{+\lambda t}-1-\lambda t\right)}{\lambda t} \approx \frac{\lambda t}{2}(\lambda t \ll 1),
$$

and

$$
\frac{n_{4}(t)}{n_{5}(t)}=\frac{n_{5}(0) \lambda t e^{-\lambda t}}{n_{5}(0) e^{-\lambda t}}=\lambda t(\text { exact })
$$

respectively. As we will see, the ratio of the number of ${ }^{74}$ As atoms present in the sample at time $t$ to the initial number of ${ }^{75}$ As atoms in the sample,

$$
\frac{n_{4}(t)}{n_{5}(0)}=\frac{n_{5}(0) \lambda t e^{-\lambda t}}{n_{5}(0)}=\lambda t e^{-\lambda t} \approx \lambda t(\lambda t \ll 1)
$$

will also be of interest because of its historic use in the post-irradiation analysis process. Generic (dimensionless) plots of these isotopic ratios and the relative errors associated with using the approximate $(\lambda t<<1)$ solutions are shown in Figures $3 \mathrm{a}$ and $3 \mathrm{~b}$, respectively, at the end of this document for $\lambda t$ values ranging from 0 to 0.250 .

\section{Historic approach used to estimate $\lambda T$}

The goal during post-irradiation radiochemical analysis of data from arsenic experiments is to estimate the value of $\lambda \mathrm{T}$ in the source region based on the isotopic abundances and ratios listed in the previous section. We will outline the historic approach here.

In most practical applications, the original arsenic sample will be explosively dispersed during the irradiation process, with the different isotopes being uniformly distributed in the debris. This will make direct measurements of the absolute number of reaction products virtually impossible; however, since the products are radioactive, the ${ }^{73} \mathrm{As} /{ }^{74} \mathrm{As}$ iso- 
topic ratio in a recovered portion of the debris can be measured spectroscopically to provide an estimate, $\mathrm{R}_{3 / 4}$, for $n_{3}(t) / n_{4}(t)$ at $t \gg \mathrm{T}$ and, following corrections for natural decay rates back to $t \sim \mathrm{T}$, an estimate, $\mathrm{R}_{3 / 4}^{\prime}$, for $n_{3}(\mathrm{~T}) / n_{4}(\mathrm{~T})$ can be obtained. If the arsenic in the original sample was the only arsenic present in the immediate environment of the experiment, then the value of $\lambda \mathrm{T}$ in the source region could easily be estimated directly from Eqn. (22) evaluated at $t=\mathrm{T}$; unfortunately, this will rarely be the case. In most experiments, there will be other materials in the immediate environment (e.g. Pb shielding), which contain trace amounts of arsenic that will also be exposed to high-energy neutrons and inseparably mixed into the debris during the irradiation process. Since this will bias the estimate for the "sample fraction" of $n_{3}(\mathrm{~T}) / n_{4}(\mathrm{~T})$, these backgrounds must somehow be factored out of $\mathrm{R}_{3 / 4}^{\prime}$ before it can be used to estimate the value of $\lambda \mathrm{T}$.

In order to factor the ${ }^{73} \mathrm{As}$ and ${ }^{74} \mathrm{As}$ background contributions out of the corrected value $\mathrm{R}_{3 / 4}^{\prime}$, we begin by breaking the pre-irradiation experimental environment up into a central (source) region (which contains the arsenic sample) surrounded by a series of sectors with multiple zones that simulate the arrangement of external materials ( $c f$. Figure 4). If we label quantities in the source region using a single subscript of " 0 " and quantities in the surrounding sectors and zones using dual subscripts of $i=1, s$ (sectors) and $j=1, z(i)$ (zones in sector $i$ ), then $\mathrm{R}_{3 / 4}^{\prime}$ can be expressed as

$$
\mathrm{R}_{3 / 4}^{\prime}=\frac{n_{3}(\mathrm{~T})(\text { sample }+ \text { bkgnd })}{n_{4}(\mathrm{~T})(\text { sample }+ \text { bkgnd })}=\frac{n_{3,0}(\mathrm{~T})+\sum_{i=1}^{s} \sum_{j=1}^{z(i)} n_{3, i j}(\mathrm{~T})}{n_{4,0}(\mathrm{~T})+\sum_{i=1}^{s} \sum_{j=1}^{z(i)} n_{4, i j}(\mathrm{~T})} .
$$

For $\lambda \mathrm{T}<<1$ (which will typically be the case), Eqns. (19) and (20) indicate that the expressions for $n_{3}(\mathrm{~T})$ and $n_{4}(\mathrm{~T})$ in Eqn. (25) reduce to

$$
n_{3}(\mathrm{~T})(\text { sample }+ \text { bkgnd }) \approx n_{5,0}(0) \frac{\left(\lambda_{0} \mathrm{~T}\right)^{2}}{2}+\sum_{i=1}^{s} \sum_{j=1}^{z(i)} n_{5, i j}(0) \frac{\left(\lambda_{i j} \mathrm{~T}\right)^{2}}{2}
$$

and

$$
n_{4}(\mathrm{~T})(\text { sample }+ \text { bkgnd }) \approx n_{5,0}(0) \lambda_{0} \mathrm{~T}+\sum_{i=1}^{s} \sum_{j=1}^{z(i)} n_{5, i j}(0) \lambda_{i j} \mathrm{~T},
$$

respectively, where $\lambda_{0} \mathrm{~T}$ is the effective value of $\lambda \mathrm{T}$ in the source region, $\lambda_{i j} \mathrm{~T} \leq \lambda_{0} \mathrm{~T}$ is the effective value of $\lambda \mathrm{T}$ in zone $j$ of external sector $i$ and $n_{5,0}(0)$ and $n_{5, i j}(0)$ (the initial number of ${ }^{75}$ As atoms in the sample and in the surrounding materials, respectively) are known or can at least be determined.

Although it can be represented in a number of different ways, the rate constant $\lambda_{0}$ in this model is essentially equal to the average neutron flux, $F_{0}$, at some characteristic point of interest, $r_{0}>0$, in the source region multiplied by the arsenic $(\mathrm{n}, 2 \mathrm{n})$ reaction cross section at the neutron energy of interest. The average neutron flux, $F_{i j}$, at a point $r_{i j}$ near the center of zone $j$ in external sector $i$ is thus related to $F_{0}$ by an equation of the form 


$$
F_{i j} \approx F_{0} \operatorname{Exp}\left\{-\mu_{i 1} \ell_{i 1}-\mu_{i 2} \ell_{i 2} \ldots-\mu_{i j}\left(\ell_{i j} / 2\right)\right\}\left(r_{0} / r_{i j}\right)^{2}=F_{0} G_{i j},
$$

where the exponential term in the geometry factor, $G_{i j}$, represents the average transmission of high-energy neutrons through each successive material leading from the source region to the center of zone $j$ and the squared term at the end is a solid angle correction. ${ }^{2}$ The value of $\lambda_{i j}$ at $r_{i j}$ will thus be related to $\lambda_{0}$ by

$$
\lambda_{i j}=F_{i j} \sigma(\mathrm{n}, 2 \mathrm{n}) \approx F_{0} G_{i j} \sigma(\mathrm{n}, 2 \mathrm{n})=\lambda_{0} G_{i j} .
$$

Using this approximate but very convenient relationship between the $\lambda_{i j}$ values and $\lambda_{0}$, we see that Eqns. (26) and (27) can be expressed as

$$
n_{3}(\mathrm{~T})(\text { sample }+ \text { bkgnd }) \approx \frac{\left(\lambda_{0} \mathrm{~T}\right)^{2}}{2}\left\{n_{5,0}(0)+\sum_{i=1}^{s} \sum_{j=1}^{z(i)} n_{5, i j}(0) G_{i j}^{2}\right\}
$$

and

$$
n_{4}(\mathrm{~T})(\text { sample }+ \text { bkgnd }) \approx \lambda_{0} \mathrm{~T}\left\{n_{5,0}(0)+\sum_{i=1}^{s} \sum_{j=1}^{z(i)} n_{5, i j}(0) G_{i j}\right\},
$$

respectively. $R_{3 / 4}^{\prime}$ can thus be written in terms of $\lambda_{0} T$ as

$$
\mathrm{R}_{3 / 4}^{\prime} \approx \frac{\lambda_{0} \mathrm{~T}}{2}\left\{\frac{n_{5,0}(0)+\sum_{i=1}^{s} \sum_{j=1}^{z(i)} n_{5, i j}(0) G_{i j}^{2}}{n_{5,0}(0)+\sum_{i=1}^{s} \sum_{j=1}^{z(i)} n_{5, i j}(0) G_{i j}}\right\} .
$$

Astute readers will note that we could simply solve Eqn. (32) for $\lambda_{0} \mathrm{~T}$ as

$$
\lambda_{0} \mathrm{~T} \approx 2 \mathrm{R}_{3 / 4}^{\prime}\left\{\frac{n_{5,0}(0)+\sum_{i=1}^{s} \sum_{j=1}^{z(i)} n_{5, i j}(0) G_{i j}}{n_{5,0}(0)+\sum_{i=1}^{s} \sum_{j=1}^{z(i)} n_{5, i j}(0) G_{i j}^{2}}\right\}
$$

and be done with the analysis at this point if we chose to do so; however, the historic approach has been to derive an estimate for $\lambda_{0} \mathrm{~T}$ using background corrected estimates for $n_{3,0}(\mathrm{~T}), n_{4,0}(\mathrm{~T})$ and/or $n_{3,0}(\mathrm{~T}) / n_{4,0}(\mathrm{~T}) \equiv \mathrm{R}_{3 / 4}^{\prime \prime}$, so we will continue by illustrating the derivations of these quantities for the sake of historical reference.

Recalling from Eqn. (24) that for $\lambda_{0} \mathrm{~T}<<1$,

\footnotetext{
${ }^{2}$ While the historic approach has been to manually estimate the geometry factors, $G_{i j}$, relating $F_{i j}$ to $F_{0}$ and $\lambda_{i j}$ to $\lambda_{0}$ in Eqns. (28) and (29), respectively, we note that a much less tedious - and almost certainly more accurate - approach might be to use detailed Monte Carlo simulations of the experiment and its immediate environment to estimate $G_{i j}$ values in regions of interest based on average flux ratios $\left(F_{i j} / F_{0}\right)$. This would allow analysts the opportunity to account for neutron backscatter by folding the energy-dependent neutron spectra in regions of interest together with the full arsenic $(n, 2 n)$ reaction cross sections.
} 


$$
\frac{n_{4,0}(\mathrm{~T})}{n_{5,0}(0)} \approx \lambda_{0} \mathrm{~T},
$$

we see that Eqn. (32) can re-expressed in terms of $n_{4,0}(\mathrm{~T})$ and $n_{5,0}(0)$ as

$$
\mathrm{R}_{3 / 4}^{\prime} \approx \frac{1}{2} \frac{n_{4,0}(\mathrm{~T})}{n_{5,0}(0)}\left\{\frac{n_{5,0}(0)+\sum_{i=1}^{s} \sum_{j=1}^{z(i)} n_{5, i j}(0) G_{i j}^{2}}{n_{5,0}(0)+\sum_{i=1}^{s} \sum_{j=1}^{z(i)} n_{5, i j}(0) G_{i j}}\right\}
$$

which allows us to generate the following estimate for $n_{4,0}(\mathrm{~T})$ :

$$
n_{4,0}(\mathrm{~T}) \approx 2 n_{5,0}(0) \mathrm{R}_{3 / 4}^{\prime}\left\{\frac{n_{5,0}(0)+\sum_{i=1}^{s} \sum_{j=1}^{z(i)} n_{5, i j}(0) G_{i j}}{n_{5,0}(0)+\sum_{i=1}^{s} \sum_{j=1}^{z(i)} n_{5, i j}(0) G_{i j}^{2}}\right\} .
$$

If we combine Eqns. (22) and (24) for $\lambda_{0} \mathrm{~T}<<1$, we see that

$$
\frac{n_{4,0}(\mathrm{~T})}{n_{5,0}(0)} \approx 2 \frac{n_{3,0}(\mathrm{~T})}{n_{4,0}(\mathrm{~T})},
$$

which allows us to generate the following estimate for $n_{3,0}(\mathrm{~T})$ :

$$
n_{3,0}(\mathrm{~T}) \approx \frac{\left(n_{4,0}(\mathrm{~T})\right)^{2}}{2 n_{5,0}(0)} \approx 2 n_{5,0}(0)\left(\mathrm{R}_{3 / 4}^{\prime}\right)^{2}\left\{\frac{n_{5,0}(0)+\sum_{i=1}^{s} \sum_{j=1}^{z(i)} n_{5, i j}(0) G_{i j}}{n_{5,0}(0)+\sum_{i=1}^{s} \sum_{j=1}^{z(i)} n_{5, i j}(0) G_{i j}^{2}}\right\}^{2} .
$$

If we combine Eqns. (36) and (38), the final (reported) estimate for the "sample fraction" of $n_{3}(\mathrm{~T}) / n_{4}(\mathrm{~T})$ thus becomes

$$
\mathrm{R}_{3 / 4}^{\prime \prime} \approx \mathrm{R}_{3 / 4}^{\prime}\left\{\frac{n_{5,0}(0)+\sum_{i=1}^{s} \sum_{j=1}^{z(i)} n_{5, i j}(0) G_{i j}}{n_{5,0}(0)+\sum_{i=1}^{s} \sum_{j=1}^{z(i)} n_{5, i j}(0) G_{i j}^{2}}\right\} \geq \mathrm{R}_{3 / 4}^{\prime} .
$$

Apart from perhaps using Eqn (22) for $\lambda_{0} \mathrm{~T}<<1$ to estimate $\lambda_{0} \mathrm{~T}$ as

$$
\lambda_{0} \mathrm{~T} \approx 2 \frac{n_{3,0}(\mathrm{~T})}{n_{4,0}(\mathrm{~T})} \approx 2 \mathrm{R}_{3 / 4}^{\prime \prime},
$$

this would complete the historical analysis from the radiochemistry perspective.

Regardless of whether one uses Eqn. (33) or its historical equivalent, Eqn. (40), to derive an estimate for $\lambda_{0} \mathrm{~T}$, the total error in the derived value will be very difficult to estimate, 
primarily due to the myriad approximations involved in factoring the ${ }^{73} \mathrm{As}$ and ${ }^{74} \mathrm{As}$ background contributions out of the measured value $\mathrm{R}_{3 / 4}^{\prime}$. Unless the experimental environment is either very simple (e.g. essentially no shielding in the immediate vicinity of the source) or very clean (e.g. no measurable traces of arsenic in nearby materials), the total error in the derived estimate for $\lambda_{0} \mathrm{~T}$ will likely be dominated by errors associated with the techniques used to do the background corrections, in particular, the historic (manual) approach used to estimate the geometry factors, $G_{i j}$, that relate the average $(\mathrm{n}, 2 \mathrm{n})$ rate constants in external sectors, $\lambda_{i j}$, to the rate constant of interest in the source region, $\lambda_{0}$. As noted earlier, Monte Carlo simulations of the experiment and its immediate environment would probably provide more accurate estimates for the $G_{i j}$ values.

\section{Summary comments}

In this report, we have provided detailed derivations for the ${ }^{73} \mathrm{As},{ }^{74} \mathrm{As}$ and ${ }^{75} \mathrm{As}$ isotopic abundances and ratios in an arsenic sample irradiated by high-energy (14 MeV) neutrons for $0 \leq t \leq \mathrm{T}$, where $\mathrm{T}$ is short compared to the natural decay times of the reaction products $\left(t_{1 / 2}\left({ }^{73} \mathrm{As}\right) \sim 80.3 \mathrm{~d}, t_{1 / 2}\left({ }^{74} \mathrm{As}\right) \sim 17.8 \mathrm{~d}\right)$. We have also outlined the historic approach used to estimate the value of $\lambda \mathrm{T}$ in the source region based on post-irradiation measurements of the ${ }^{73} \mathrm{As} /{ }^{74} \mathrm{As}$ isotopic ratio. It is hoped that this document will clear up several misconceptions known to be floating around with regard to the exact formulas for the isotopic abundances and ratios and prove useful as a primer to those working on data from the Nuclear Test Program and/or NIF experiments. Finally, we note that the procedures used to estimate the neutron flux and/or yield from a source based on derived $\lambda \mathrm{T}$ values have not been discussed here as they are beyond the intended scope of this report. 


\section{Appendix A}

This Appendix provides derivations of the relative isotopic abundances and ratios for an arsenic radiochemical detector for situations in which the reaction rates $\lambda_{1}$ and $\lambda_{2}$ cannot be assumed to be equal (e.g. Monte Carlo simulation in which the full neutron spectrum is taken into account). The development parallels that presented above.

If the reaction rates $\lambda_{1}$ and $\lambda_{2}$ are treated as distinct quantities, then the rate equations for $n_{5}\left({ }^{75} \mathrm{As}\right), n_{4}\left({ }^{74} \mathrm{As}\right)$ and $n_{3}\left({ }^{73} \mathrm{As}\right)$ become

$$
\begin{gathered}
\frac{d n_{5}}{d t}=-\lambda_{1} n_{5} \\
\frac{d n_{4}}{d t}=-\lambda_{2} n_{4}+\lambda_{1} n_{5}
\end{gathered}
$$

and

$$
\frac{d n_{3}}{d t}=+\lambda_{2} n_{4}
$$

respectively, where we have again neglected the comparatively slow natural decay rates of ${ }^{74}$ As $\left(t_{1 / 2} \sim 17.8 \mathrm{~d}\right)$ and ${ }^{73} \mathrm{As}\left(t_{1 / 2} \sim 80.3 \mathrm{~d}\right)$.

Using the same techniques as before, the solutions for the isotopic abundances of ${ }^{73} \mathrm{As}$, ${ }^{74}$ As and ${ }^{75}$ As for arbitrary times $0 \leq t \leq \mathrm{T}$ can be shown to be

$$
\begin{gathered}
n_{3}(t)=n_{5}(0)\left\{1-\frac{\lambda_{1}}{\left(\lambda_{2}-\lambda_{1}\right)}\left(e^{-\lambda_{1} t}-e^{-\lambda_{2} t}\right)-e^{-\lambda_{1} t}\right\} \\
\approx n_{5}(0) \frac{\lambda_{1} \lambda_{2} t^{2}}{2}\left(\lambda_{1} t, \lambda_{2} t<<1\right), \\
n_{4}(t)=n_{5}(0)\left\{\frac{\lambda_{1}}{\left(\lambda_{2}-\lambda_{1}\right)}\left(e^{-\lambda_{1} t}-e^{-\lambda_{2} t}\right)\right\} \approx n_{5}(0) \lambda_{1} t\left(\lambda_{1} t, \lambda_{2} t<<1\right)
\end{gathered}
$$

and

$$
n_{5}(t)=n_{5}(0) e^{-\lambda_{1} t} \approx n_{5}(0)\left(1-\lambda_{1} t\right)\left(\lambda_{1} t<<1\right),
$$

respectively. Note that $n_{3}(t)+n_{4}(t)+n_{5}(t)=n_{5}(0)$ for $0 \leq t \leq \mathrm{T}$ as we would expect (i.e. the exact solutions are self consistent and the approximate solutions for $\lambda_{1} t, \lambda_{2} t<<1$ are self consistent to within terms $\sim \lambda_{1} \lambda_{2} t^{2}$ ). Note also that, while the exact solutions for $n_{3}(t)$ and $n_{4}(t)$ appear to be quite different in form from their counterparts derived assuming that $\lambda_{1} \sim \lambda_{2} \equiv \lambda$ (i.e. Eqns (19) and (20), respectively), the approximate solutions are actually very similar in form.

Using the solution set summarized above, the ${ }^{73} \mathrm{As} /{ }^{74} \mathrm{As},{ }^{74} \mathrm{As} /{ }^{75} \mathrm{As}$ and ${ }^{74} \mathrm{As} /\left(\right.$ initial $\left.{ }^{75} \mathrm{As}\right)$ isotopic ratios for arbitrary times $0 \leq t \leq \mathrm{T}$ can be shown to be 


$$
\begin{gathered}
\frac{n_{3}(t)}{n_{4}(t)}=\frac{\lambda_{2}\left(1-e^{-\lambda_{1} t}\right)-\lambda_{1}\left(1-e^{-\lambda_{2} t}\right)}{\lambda_{1}\left(e^{-\lambda_{1} t}-e^{-\lambda_{2} t}\right)} \approx \frac{\lambda_{2} t}{2}\left(\lambda_{1} t, \lambda_{2} t<1\right), \\
\frac{n_{4}(t)}{n_{5}(t)}=\frac{\lambda_{1} e^{+\lambda_{1} t}}{\left(\lambda_{2}-\lambda_{1}\right)}\left(e^{-\lambda_{1} t}-e^{-\lambda_{2} t}\right) \approx \lambda_{1} t\left(\lambda_{1} t, \lambda_{2} t<<1\right)
\end{gathered}
$$

and

$$
\frac{n_{4}(t)}{n_{5}(0)}=\frac{\lambda_{1}}{\left(\lambda_{2}-\lambda_{1}\right)}\left(e^{-\lambda_{1} t}-e^{-\lambda_{2} t}\right) \approx \lambda_{1} t\left(\lambda_{1} t, \lambda_{2} t<1\right),
$$

respectively. Note that, while the exact ratios for $n_{3}(t) / n_{4}(t), n_{4}(t) / n_{5}(t)$ and $n_{4}(t) / n_{5}(0)$ appear to be quite different from their counterparts derived assuming that $\lambda_{1} \sim \lambda_{2} \equiv \lambda$ (i.e. Eqns (22), (23) and (24), respectively), the approximate solutions for the ratios are again very similar in form.

The radiochemical analysis of debris recovered from an experiment would again begin with spectroscopic measurements to estimate $n_{3}(t) / n_{4}(t) \equiv \mathrm{R}_{3 / 4}$ at $t>>\mathrm{T}$, which would then be corrected for the natural decay rates of ${ }^{73} \mathrm{As}$ and ${ }^{74} \mathrm{As}$ back to $t \sim \mathrm{T}$ in order to obtain an estimate for $n_{3}(\mathrm{~T}) / n_{4}(\mathrm{~T}) \equiv \mathrm{R}_{3 / 4}^{\prime}$. Using a notation scheme similar to that used before, $\mathrm{R}_{3 / 4}^{\prime}$ can be expressed as

$$
\mathrm{R}_{3 / 4}^{\prime}=\frac{n_{3}(\mathrm{~T})(\text { sample }+ \text { bkgnd })}{n_{4}(\mathrm{~T})(\text { sample }+ \text { bkgnd })}=\frac{n_{3,0}(\mathrm{~T})+\sum_{i=1}^{s} \sum_{j=1}^{z(i)} n_{3, i j}(\mathrm{~T})}{n_{4,0}(\mathrm{~T})+\sum_{i=1}^{s} \sum_{j=1}^{z(i)} n_{4, i j}(\mathrm{~T})} .
$$

For $\lambda_{1} t, \lambda_{2} t<<1$, Eqns. (A4) and (A5) indicate that the expressions for $n_{3}(\mathrm{~T})$ and $n_{4}(\mathrm{~T})$ in Eqn. (A10) reduce to

$$
n_{3}(\mathrm{~T})(\text { sample }+ \text { bkgnd }) \approx n_{5,0}(0) \frac{\lambda_{1,0} \lambda_{2,0} \mathrm{~T}^{2}}{2}+\sum_{i=1}^{s} \sum_{j=1}^{z(i)} n_{5, i j}(0) \frac{\lambda_{1, i j} \lambda_{2, i j} \mathrm{~T}^{2}}{2}
$$

and

$$
n_{4}(\mathrm{~T})(\text { sample }+ \text { bkgnd }) \approx n_{5,0}(0) \lambda_{1,0} \mathrm{~T}+\sum_{i=1}^{s} \sum_{j=1}^{z(i)} n_{5, i j}(0) \lambda_{1, i j} \mathrm{~T},
$$

respectively, where $\lambda_{1,0} \mathrm{~T}$ and $\lambda_{2,0} \mathrm{~T}$ are the effective values of $\lambda_{1} \mathrm{~T}$ and $\lambda_{2} \mathrm{~T}$ in the source region, $\lambda_{1, i j} \mathrm{~T} \leq \lambda_{1,0} \mathrm{~T}$ and $\lambda_{2, i j} \mathrm{~T} \leq \lambda_{2,0} \mathrm{~T}$ are the effective values of $\lambda_{1} \mathrm{~T}$ and $\lambda_{2} \mathrm{~T}$ in zone $j$ of external sector $i$ and $n_{5,0}(0)$ and $n_{5, i j}(0)$ (the initial number of ${ }^{75}$ As atoms in the sample and in the surrounding materials, respectively) are known or can be determined.

If we relate the $\lambda_{1, i j}$ and $\lambda_{2, i j}$ values in the external zones and sectors to $\lambda_{1,0}$ and $\lambda_{2,0}$ using geometry factors, $G_{1, i j}$ and $G_{2, i j}$, analogous to those used before (cf. Eqn. (29)), we see that Eqns. (A11) and (A12) can be expressed as 


$$
n_{3}(\mathrm{~T})(\text { sample }+ \text { bkgnd }) \approx \frac{\lambda_{1,0} \lambda_{2,0} \mathrm{~T}^{2}}{2}\left\{n_{5,0}(0)+\sum_{i=1}^{s} \sum_{j=1}^{z(i)} n_{5, i j}(0) G_{1, i j} G_{2, i j}\right\}
$$

and

$$
n_{4}(\mathrm{~T})(\text { sample }+ \text { bkgnd }) \approx \lambda_{1,0} \mathrm{~T}\left\{n_{5,0}(0)+\sum_{i=1}^{s} \sum_{j=1}^{z(i)} n_{5, i j}(0) G_{1, i j}\right\},
$$

respectively. $R_{3 / 4}^{\prime}$ can thus be written in terms of $\lambda_{2,0} T$ as

$$
\mathrm{R}_{3 / 4}^{\prime} \approx \frac{\lambda_{2,0} \mathrm{~T}}{2}\left\{\frac{n_{5,0}(0)+\sum_{i=1}^{s} \sum_{j=1}^{z(i)} n_{5, i j}(0) G_{1, i j} G_{2, i j}}{n_{5,0}(0)+\sum_{i=1}^{s} \sum_{j=1}^{z(i)} n_{5, i j}(0) G_{1, i j}}\right\},
$$

which can be solved for $\lambda_{2,0} \mathrm{~T}$ as

$$
\lambda_{2,0} \mathrm{~T} \approx 2 \mathrm{R}_{3 / 4}^{\prime}\left\{\frac{n_{5,0}(0)+\sum_{i=1}^{s} \sum_{j=1}^{z(i)} n_{5, i j}(0) G_{1, i j}}{n_{5,0}(0)+\sum_{i=1}^{s} \sum_{j=1}^{z(i)} n_{5, i j}(0) G_{1, i j} G_{2, i j}}\right\} .
$$

Note that, since $n_{4}(\mathrm{~T}) / n_{5}(0)$ depends on $\lambda_{1,0}$ rather than $\lambda_{2,0}$ for $\lambda_{1} t, \lambda_{2} t<<1$ (cf. Eqn. (A9)), the analysis procedure would, of necessity, diverge from the historic approach at this point (i.e. we would need to find an alternate way to estimate $\lambda_{1,0} \mathrm{~T}$ ).

The best way to estimate $\lambda_{1,0} \mathrm{~T}$ might be to use mass spectroscopy to estimate $n_{4}(t) / n_{5}(t)$ $\equiv \mathrm{R}_{4 / 5}$ at $t>>\mathrm{T}$ and then correct for the natural decay rate of ${ }^{74} \mathrm{As}$ back to $t \sim \mathrm{T}$ in order to obtain an estimate for $n_{4}(\mathrm{~T}) / n_{5}(\mathrm{~T}) \equiv \mathrm{R}_{4 / 5}^{\prime}:^{3}$

$$
\mathrm{R}_{4 / 5}^{\prime}=\frac{n_{4}(\mathrm{~T})(\text { sample }+ \text { bkgnd })}{n_{5}(\mathrm{~T})(\text { sample }+ \text { bkgnd })}=\frac{n_{4,0}(\mathrm{~T})+\sum_{i=1}^{s} \sum_{j=1}^{z(i)} n_{4, i j}(\mathrm{~T})}{n_{5,0}(\mathrm{~T})+\sum_{i=1}^{s} \sum_{j=1}^{z(i)} n_{5, i j}(\mathrm{~T})} .
$$

For $\lambda_{1} t, \lambda_{2} t<<1$, Eqns. (A5) and (A6) indicate that the expressions for $n_{4}(\mathrm{~T})$ and $n_{5}(\mathrm{~T})$ in Eqn. (A17) reduce to

$$
n_{4}(\mathrm{~T})(\text { sample }+ \text { bkgnd }) \approx n_{5,0}(0) \lambda_{1,0} \mathrm{~T}+\sum_{i=1}^{s} \sum_{j=1}^{z(i)} n_{5, i j}(0) \lambda_{1, i j} \mathrm{~T}
$$

and

\footnotetext{
${ }^{3}$ Although not traditionally done, this might also be a good way to obtain a second (independent) estimate for $\lambda_{0} \mathrm{~T}$ in the historic analysis procedure where one tacitly assumes $\lambda_{1} \sim \lambda_{2} \equiv \lambda$.
} 


$$
n_{5}(\mathrm{~T})(\text { sample }+ \text { bkgnd }) \approx n_{5,0}(0)\left(1-\lambda_{1,0} \mathrm{~T}\right)+\sum_{i=1}^{s} \sum_{j=1}^{z(i)} n_{5, i j}(0)\left(1-\lambda_{1, i j} \mathrm{~T}\right),
$$

respectively, which can be re-expressed in terms of the geometry factors $G_{1, i j}$ as

$$
n_{4}(\mathrm{~T})(\text { sample }+ \text { bkgnd }) \approx \lambda_{1,0} \mathrm{~T}\left\{n_{5,0}(0)+\sum_{i=1}^{s} \sum_{j=1}^{z(i)} n_{5, i j}(0) G_{1, i j}\right\}
$$

and

$$
\begin{aligned}
n_{5}(\mathrm{~T})(\text { sample }+ \text { bkgnd }) \approx & \left\{n_{5,0}(0)+\sum_{i=1}^{s} \sum_{j=1}^{z(i)} n_{5, i j}(0)\right\} \\
& -\lambda_{1,0} \mathrm{~T}\left\{n_{5,0}(0)+\sum_{i=1}^{s} \sum_{j=1}^{z(i)} n_{5, i j}(0) G_{1, i j}\right\} .
\end{aligned}
$$

$\mathrm{R}_{4 / 5}^{\prime}$ can thus be written in terms of $\lambda_{1,0} \mathrm{~T}$ as

$$
\mathrm{R}_{4 / 5}^{\prime} \approx \frac{\lambda_{1,0} \mathrm{~T}\left\{n_{5,0}(0)+\sum_{i=1}^{s} \sum_{j=1}^{z(i)} n_{5, i j}(0) G_{1, i j}\right\}}{\left\{n_{5,0}(0)+\sum_{i=1}^{s} \sum_{j=1}^{z(i)} n_{5, i j}(0)\right\}-\lambda_{1,0} \mathrm{~T}\left\{n_{5,0}(0)+\sum_{i=1}^{s} \sum_{j=1}^{z(i)} n_{5, i j}(0) G_{1, i j}\right\}},
$$

which can be solved for $\lambda_{1,0} \mathrm{~T}$ as

$$
\lambda_{1,0} \mathrm{~T} \approx \frac{\mathrm{R}_{3 / 4}^{\prime}}{\left(1-\mathrm{R}_{3 / 4}^{\prime}\right)}\left\{\frac{n_{5,0}(0)+\sum_{i=1}^{s} \sum_{j=1}^{z(i)} n_{5, i j}(0)}{n_{5,0}(0)+\sum_{i=1}^{s} \sum_{j=1}^{z(i)} n_{5, i j}(0) G_{1, i j}}\right\} .
$$

Having derived independent estimates for both $\lambda_{1,0} \mathrm{~T}$ and $\lambda_{2,0} \mathrm{~T}$, this would complete the analysis from the radiochemistry perspective. 
W. E. Parker \& J. M. Hall, LLNL-TR-435033 (LLNL, 2010)

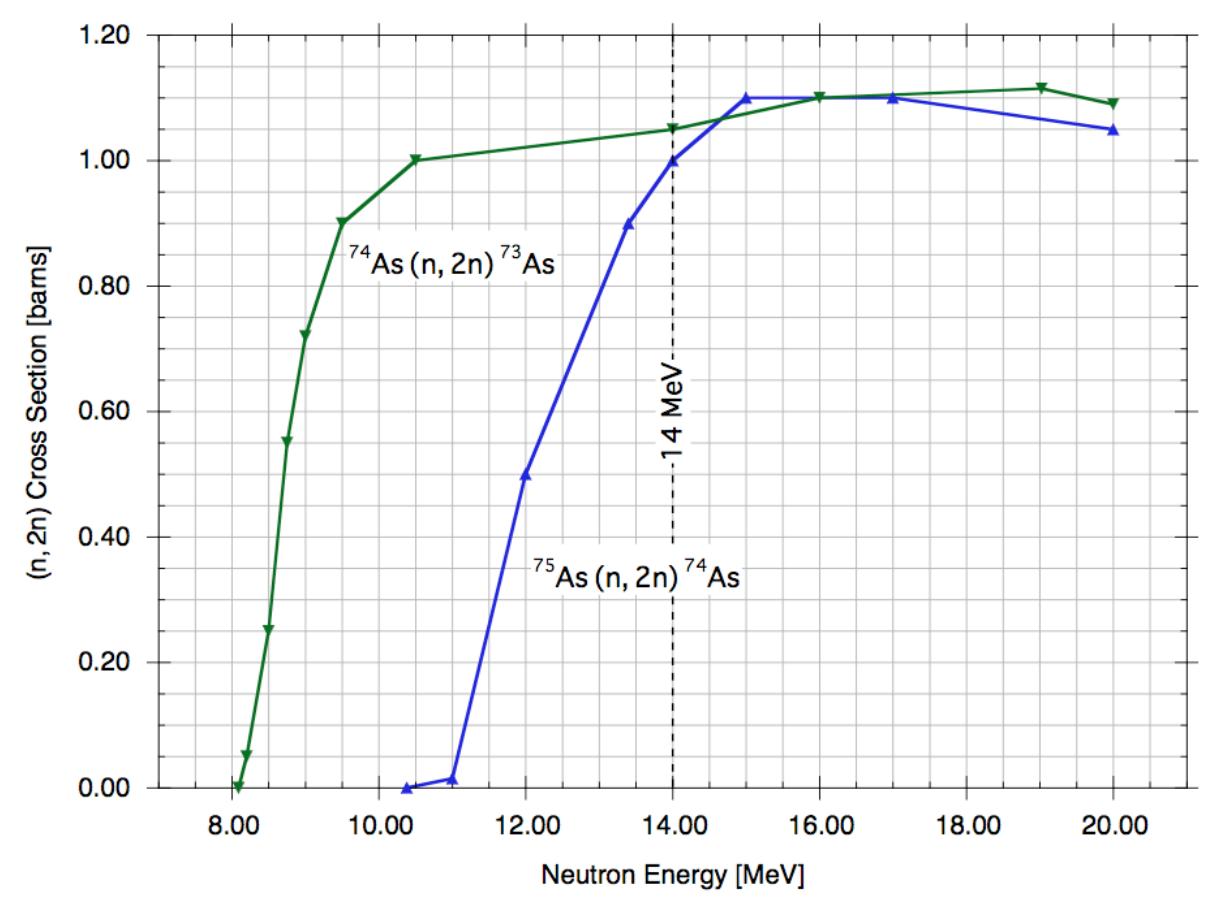

Figure 1: Arsenic (n,2n) reaction cross section data [ENDL99]. For DT fusion neutrons $(14 \mathrm{MeV})$, the $(\mathrm{n}, 2 \mathrm{n})$ reaction cross sections for ${ }^{75} \mathrm{As}$ and ${ }^{74} \mathrm{As}$ are very similar (i.e. $\lambda_{1} \sim$ $\left.\lambda_{2} \equiv \lambda\right)$; however, these rates can differ significantly at lower neutron energies.

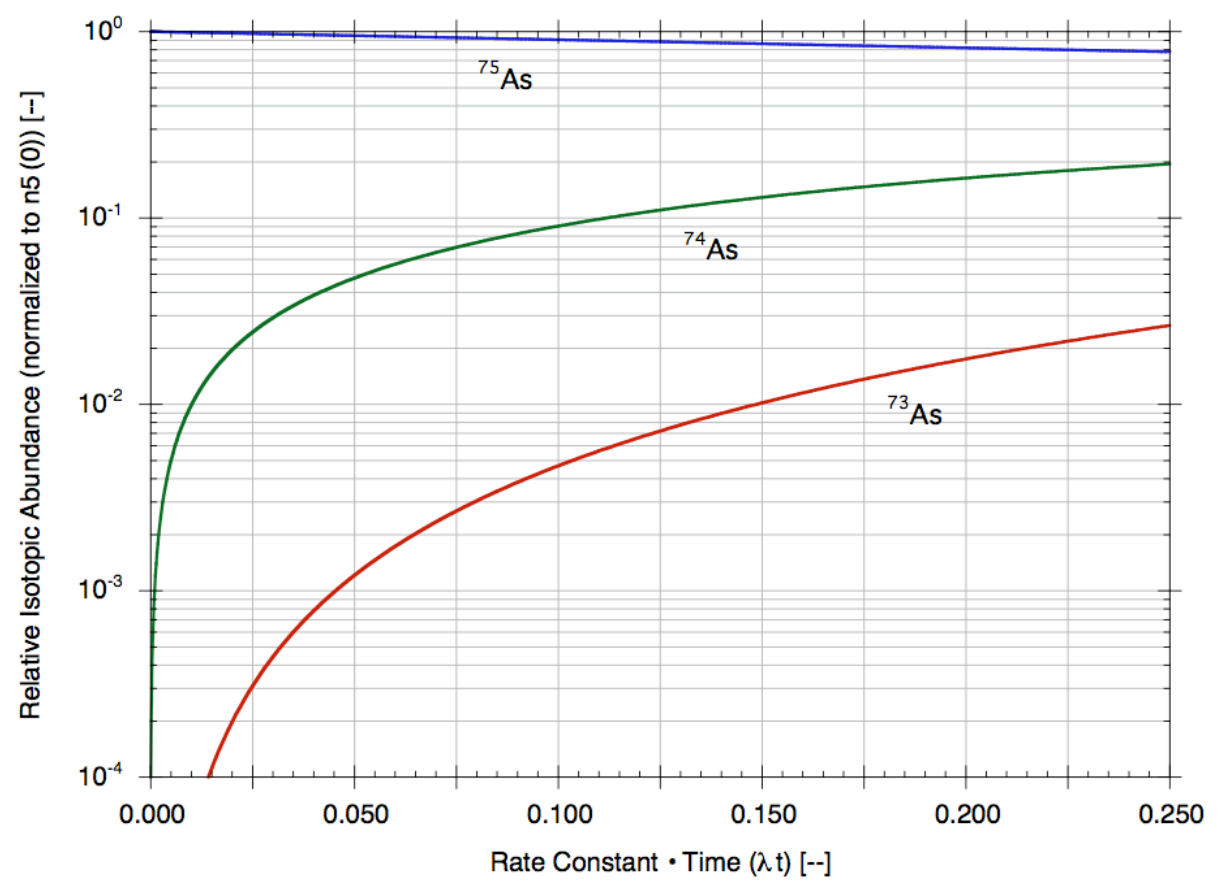

Figure 2a: Dimensionless plots of exact solutions for ${ }^{73} \mathrm{As},{ }^{74} \mathrm{As}$ and ${ }^{75} \mathrm{As}$ isotopic abundances based on Eqns. (19), (20) and (21), respectively, for $0 \leq \lambda t \leq 0.250$. For $\lambda t<<1$, the curves scale as $(\lambda t)^{2} / 2\left({ }^{73} \mathrm{As}\right), \lambda t\left({ }^{74} \mathrm{As}\right)$ and $1-\lambda t\left({ }^{75} \mathrm{As}\right)$. 
W. E. Parker \& J. M. Hall, LLNL-TR-435033 (LLNL, 2010)



Figure 2b: Relative errors associated with using the approximate $(\lambda t<<1)$ solutions for the ${ }^{73} \mathrm{As},{ }^{74} \mathrm{As}$ and ${ }^{75} \mathrm{As}$ isotopic abundances for $0 \leq \lambda t \leq 0.250$.

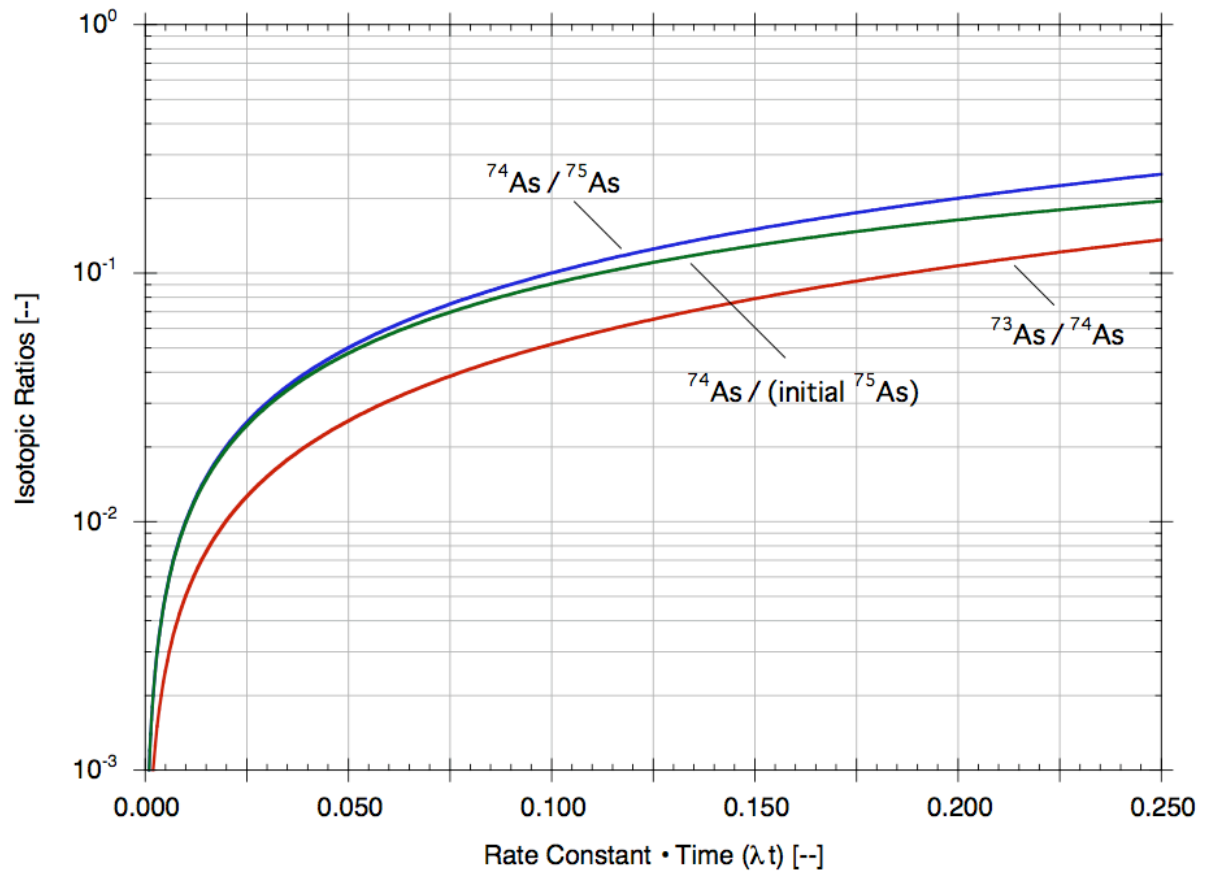

Figure 3a: Dimensionless plots of ${ }^{73} \mathrm{As} /{ }^{74} \mathrm{As},{ }^{74} \mathrm{As} /{ }^{75} \mathrm{As}$ and ${ }^{74} \mathrm{As} /\left(\right.$ initial ${ }^{75} \mathrm{As}$ ) isotopic ratios based on Eqns. (22), (23) and (24), respectively, for $0 \leq \lambda t \leq 0.250$. For $\lambda t<<1$, the curves scale as $\lambda t / 2\left({ }^{73} \mathrm{As} /{ }^{74} \mathrm{As}\right)$ and $\lambda t\left({ }^{74} \mathrm{As} /{ }^{75} \mathrm{As}\right.$ and ${ }^{74} \mathrm{As} /\left(\right.$ initial $\left.\left.{ }^{75} \mathrm{As}\right)\right)$. 
W. E. Parker \& J. M. Hall, LLNL-TR-435033 (LLNL, 2010)



Figure 3b: Relative errors associated with using the approximate $(\lambda t<<1)$ solutions for the ${ }^{73} \mathrm{As} /{ }^{74} \mathrm{As},{ }^{74} \mathrm{As} /{ }^{75} \mathrm{As}$ and ${ }^{74} \mathrm{As} /\left(\right.$ initial $\left.{ }^{75} \mathrm{As}\right)$ isotopic ratios for $0 \leq \lambda t \leq 0.250$.

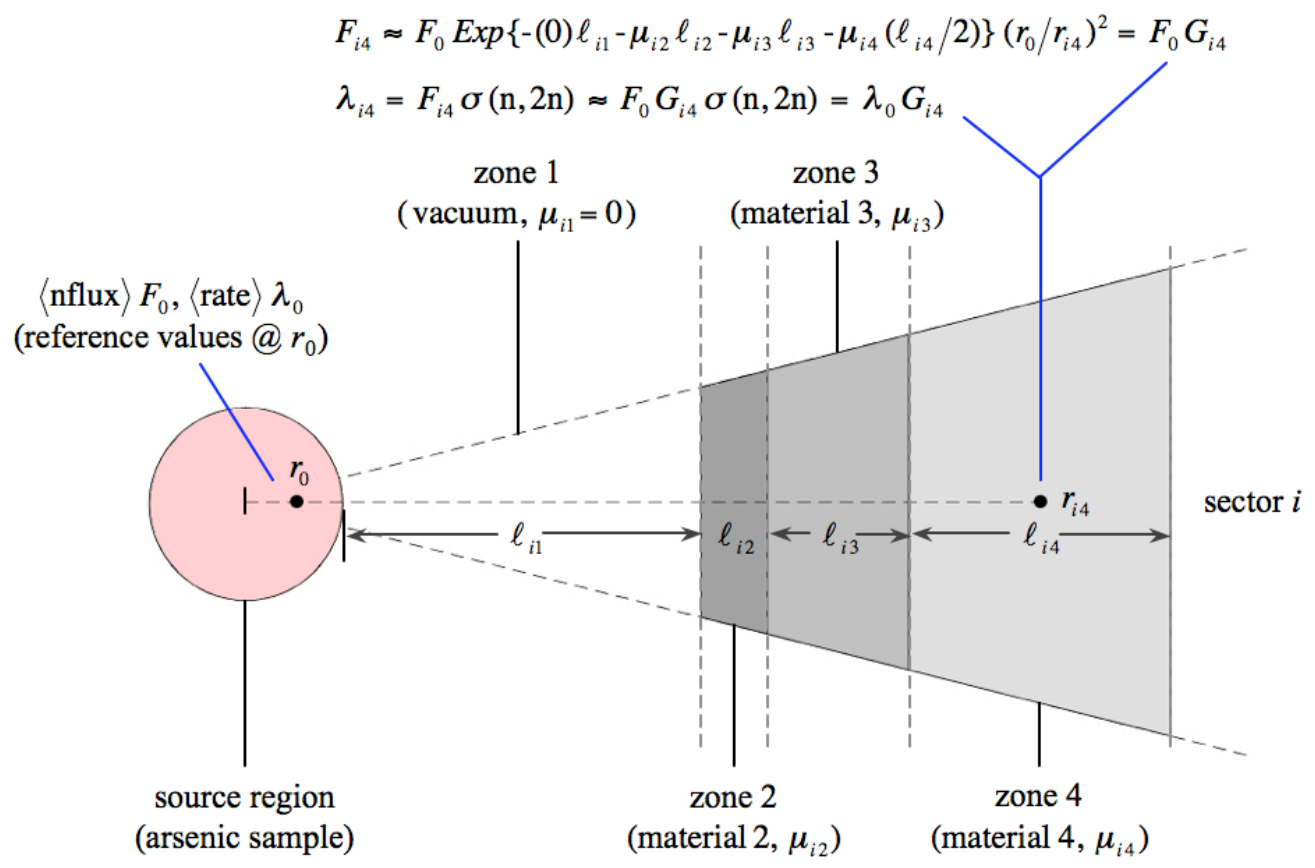

Figure 4: Generic example illustrating how the pre-irradiation experimental environment can be modeled as a central (source) region surrounded by a series of sectors with multiple zones in order to calculate the geometry factors, $G_{i j}$, that relate the effective $(\mathrm{n}, 2 \mathrm{n})$ rates in external sectors, $\lambda_{i j}$, to the reference value in the source region, $\lambda_{0}$. 